\title{
DETERMINING THE PHASE OF LINE OBJECTS BY MEASURING THEIR INTENSITY IN DARK FIELD AND BRIGHT FIELD ILLUMINATION*
}

\author{
David A. ANSLEY \\ Electrical and Computer Engineering Department. The University of Michigan, Ann Arbor, Michigan 48107. USA \\ Received 16 Iebruary 1973 \\ Revised manuseript received 8 April 1973
}

\begin{abstract}
The in situ phase of a transparent line object can be calculated from measurements of its bright field and dark field intensity. If the sampling aperture is a slit, the background can be subtracted out for increased accuracy. This method can measure phase over a range of approximately $3^{\circ}$ to $180^{\circ}$. The theory has been verified by means of an indirect experiment.
\end{abstract}

\section{Introduction}

The phase of transparent objects can be accurately measured using a Zernike three-beam interferometer [1] if the objects are larger than approximately $1 \mathrm{~mm}$. For smaller objects, the alignment of the mask becomes increasingly difficult. If the phase object is recorded on photographic film, the Eberhard effect [2] must be taken into consideration. Therefore indirect measurement of the phase by using a larger object will yield a value that is smaller than the actual phase of the submillimeter object.

In the following section we describe a method for measuring the in situ phase of small transparent objects. The method works particularly well for line objects.

\section{Theory}

Given a phase object of the form:

$1+\exp [\mathrm{i} \Phi(x)]$.

If we assume the function is a line of width $a$ centered on the line $x=0$, the Fourier transform of eq. (1) in

\footnotetext{
* Work supported by the National Science Foundation Grant No. GK-31635.
}

rectangular coordinates is

$$
\begin{aligned}
& D^{2}(2 \pi)^{-2} \operatorname{sinc}\left(\omega_{x} D / 2\right) \operatorname{sinc}\left(\omega_{y} D / 2\right) \\
& \quad+D a(2 \pi)^{-2} \operatorname{sinc}\left(\omega_{x} a / 2\right)\left(\mathrm{e}^{\mathrm{j} \Phi}-1\right) \operatorname{sinc}\left(\omega_{y} D / 2\right),(2)
\end{aligned}
$$

where $D$ is the dimension of the object aperture and $\operatorname{sinc}(\zeta)=\sin (\zeta) / \zeta$.

We can put in a semi-infinite mask that blocks the 3 rd and 4th quadrants of the transform plane and extends a distance $\epsilon$ into the 1 st and 2 nd quadrants. If $D \gg a$ and $\epsilon$ is large compared to $\lambda F / D$ but small compared to $\lambda F / a$, where $F$ is the focal length of the transform lens, the first term of (2) is effectively blocked while part of the second term is inverse Fourier transformed.

It is well known that for $x=0$ the Fourier kernels equal 1 and

$$
\lim _{\epsilon \rightarrow 0} \int_{\epsilon}^{\infty} \int \operatorname{Da}(2 \pi)^{-2} \operatorname{sinc}\left(\omega_{x} a / 2\right)
$$

$$
X \operatorname{sinc}\left(\omega_{y} D / 2\right) \mathrm{d} \omega_{x} \mathrm{~d} \omega_{y}=1 / 2 \text {. }
$$

Therefore the ratio of the amplitude of the image of the dark field illumination to bright field is:

$A_{\text {dark }}(0) / A_{\text {bright }}(0)=\mathrm{i} \sin (\Phi / 2) \exp (\mathrm{i} \Phi / 2)$.

The ratio of the line intensity in dark field illumina- 


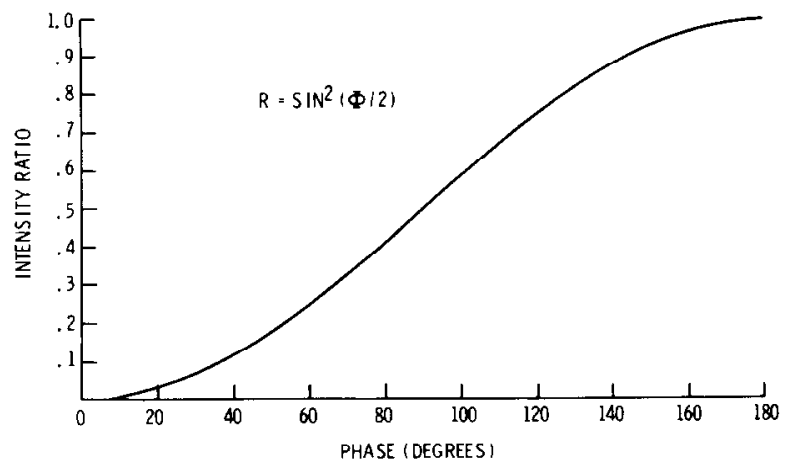

Iïg. 1. Intensity ratio versus phase.

tion to bright field is:

$R=I_{\text {dark }}(0) / I_{\text {bright }}(0)=\sin ^{2}(\Phi / 2)$.

Thus the phase of the line can be determined from eq. (5). Eq. (5) is plotted in fig. 1.

Sources of error are

(i) $\epsilon$ not zero, thus eq. (3) does not equal $1 / 2$;

(ii) scattered light.

We will now analyze the error due to scattered light. Let $B$ be the noise background intensity and $M$ the measured line intensity. The total detected intensity is

$M=\left|\mathrm{i} \sin (\Phi / 2) \exp (\mathrm{i} \Phi / 2)+B^{1 / 2} \exp (\mathrm{i} \Psi)\right|^{2}$.

Expanding eq. (6) we get:

$M=\sin ^{2}(\Phi / 2)-2 B^{1 / 2} \sin (\Phi / 2) \sin (\Phi / 2-\Psi)+B$.

If we sample over a "long" length of line, i.e., use a slit, then the expected valuc of $\sin (\Phi / 2-\Psi)=0$. Therefore we can obtain an accurate estimate of $\Phi$ simply by subtracting the background intensity $B$.

The sensitivity of the method decreases for small values of $\Phi$

$S=\frac{\mathrm{d} \Phi}{\mathrm{d} R}=\frac{1}{\sin (\Phi / 2) \cos (\Phi / 2)}$,

so $\Phi=2^{\circ}$ or $3^{\circ}$ is probably the practical limit of this method. Eq. (8) is plotted in fig. 2.

Greater sensitivity by a factor of 4 could be obtained by using a wire of thickness $2 \epsilon$ instead of the semi-infinite half plane.

The restriction that the line object be centered on the line $x=0$ is, of course, to simplify the mathematics. In practice, the line object can be located anywhere in the $x y$ plane. If the line object is rotated the semi-

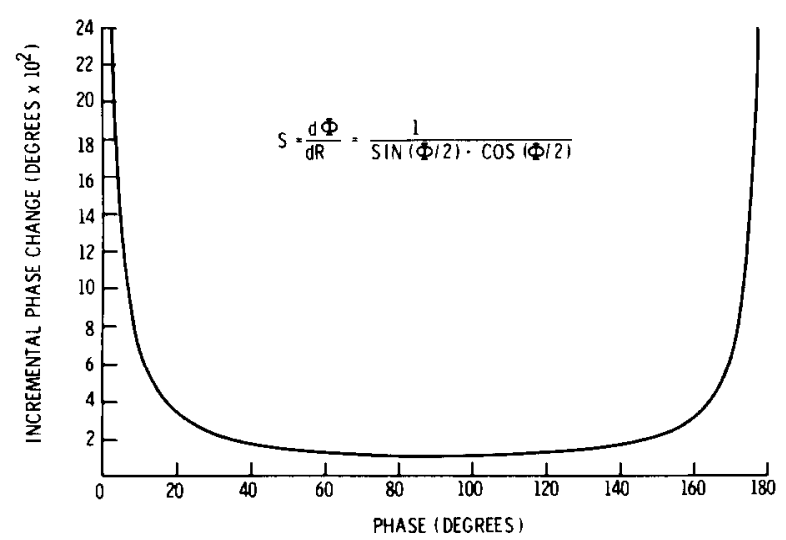

Fig. 2. Incremental phase change versus phase.

infinite mask must be rotated to the same angle.

\section{Discussion}

This method was used to measure the phase of several 40 micron wide lines [3]. The lines were recorded onto Agfa 10E70 film. Develupment was in Kodak D76 for 5 minutes at approximately $20^{\circ} \mathrm{C}$. The films were then bleached in a potassium ferrocyanide and sodium carbonate solution. The measured phase of the lines ranged from approximately $3^{\circ}$ to $45^{\circ}$. The accuracy of the measurements was indirectly confirmed by comparing the experimental and computer simulated images of a defocused phase line object. Using the measured value for the phase, the shape of the computer simulated image compared closely with the experimental image.

Complex structure was not noticed in the dark field image although the procedure of blocking the undiffracted light is similar to a Foucault knife edge test. There are two reasons for this: (a) the optics in the experiment were essentially diffraction limited and (b) the value of $c$ was somewhat greater than the value that would be used for a Foucault knife edge test.

\section{References}

[1] F. Zernike, J. Opt. Soc. Am. 40 (1950) 326.

[2] Kodak Plates and Films for Science and Industry, p. 15.

[3] D.A. Ansley, Ph.D. Dissertation, University of Michigan (1973). 